\title{
A computational aspect of the Lebesgue differentiation theorem
}

\author{
NOOPUR PATHAK
}

\begin{abstract}
Given an $L_{1}$-computable function, $f$, we identify a canonical representative of the equivalence class of $f$, where $f$ and $g$ are equivalent if and only if $\int|f-g|$ is zero. Using this representative, we prove a modified version of the Lebesgue Differentiation Theorem. Our theorem is stated in terms of Martin-Löf random points in Euclidean space.
\end{abstract}

2000 Mathematics Subject Classification 03D80 (primary); 26A24 (secondary)

Keywords: Lebesgue Differentiation Theorem, Martin-Lof Randomness, algorithmic randomness

\section{Introduction}

The Lebesgue Differentiation Theorem is a fundamental theorem in measure theory which generalizes the fundamental theorem of calculus. The Lebesgue Differentiation Theorem states that given $f \in L_{1}\left([0,1]^{d}\right)$,

$$
f(x)=\lim _{Q \backslash x} \frac{\int_{Q} f}{\mu(Q)}
$$

for almost every $x$ where $Q$ is a cube in $[0,1]^{d}$ containing $x$. In this paper, we look at the theorem in the context of computability theory. A proof of the Lebesgue Differentiation theorem can be found in the book of Wheeden-Zygmund [6] (p. 101109 ) and with some work we can modify this proof for $L_{1}$-computable functions which are defined in Theorem 2.1. The final result we obtain will be a modified version of the Lebesgue Differentiation Theorem and will hold for all $x$ which are Martin-Löf random. Due to the nature of Lebesgue integration, rather than working with actual functions $f$, it will be more useful to work with canonical representatives of $f$ based on the equivalence relation

$$
f \sim g \Leftrightarrow\|f-g\|_{1}=0 .
$$

In this paper, we will prove such a canonical representative exists, and is well defined. Eventually, upon using some ideas from the original proof and creating some new 
tests for randomness, we will prove Theorem 5.1. A useful reference on computable analysis is given by Pour-El and Richards [4] and many of the ideas and terms in this paper come from that book.

This paper is a revised version of a report completed for an undergraduate course on Computability and Randomness. This course was taken in the MASS program at the Pennsylvania State University in the Fall of 2007. The author thanks Stephen Simpson for his guidance and support, without which this paper would not be possible.

The referee has pointed out a result of Demuth [2] which says approximately the following: any computable function with bounded variation is differentiable at the Martin-Löf random reals. Due to notational and other difficulties, we have not been able to determine the exact content of Demuth's result and to what extent it may overlap with out results.

\section{Some Notation and Definitions}

The functions we consider in this paper will be real-valued and Lebesgue measurable with measure $\mu$ on the unit cube $[0,1]^{d} \subset \mathbb{R}^{d}$. Here $d$ is a fixed positive integer. Our short description of this will be $f \in L_{1}\left([0,1]^{d}\right)$. Our norm will be the $L_{1}$-norm which is defined by

$$
\|f\|_{1}=\int_{[0,1]^{d}}|f|=\int_{x \in[0,1]^{d}}|f(x)| d \mu(x) .
$$

Note that $L_{1}$ functions have finite Lebesgue integral.

The following definition was provided by Pour-El and Richards [4].

Definition 2.1 A function $f \in L_{1}\left([0,1]^{d}\right)$ is called $L_{1}$-computable if there exists a computable sequence of polynomials $f_{n} \in \mathbb{Q}[x]$ such that for all $n$

$$
\left\|f-f_{n}\right\|_{1}<\frac{1}{2^{n}}
$$

For future reference, note that we can easily find a computable sequence of rational numbers $\left(D_{n}\right)$, depending on $f_{n}$, where $D_{n}$ is an upper bound of the maximum gradient of each $f_{n}, \max _{x}\left\{\left|\nabla f_{n}(x)\right|: x \in[0,1]^{d}\right\}$

Definition 2.2 Let $f$ be an $L_{1}$ function and let $Q$ denote a rational cube (that is, the coordinates of the vertices of $Q$ are rational). Given $x$, we consider those $Q$ containing 
$x$ with edges parallel to the coordinate axes. Then, the indefinite integral of $f$ is said to be differentiable at $x$ if

$$
\lim _{Q \searrow x} \frac{\int_{Q} f}{\mu(Q)}
$$

exists.

Lemma 2.3 Given a rational cube $Q \subseteq[0,1]^{d}$ and an $L_{1}$-computable function $f$, we can effectively find the computable real number $\int_{Q} f$.

Proof To show that $\int_{Q} f$ exists and is computable we need to find a recursive sequence of rational numbers, $\left(c_{n}\right)$ such that

$$
\left|c_{n}-\int_{Q} f\right|<\frac{1}{2^{n}}
$$

for all $n$. By Theorem 2.1, there exists a computable sequence of polynomials with rational coefficients, $f_{n}$, such that

$$
\left\|f-f_{n}\right\|_{1} \leq \frac{1}{2^{n}}
$$

We want to say that $c_{n}=\int_{Q} f_{n}$. First we show that $\int_{Q} f_{n}$ is rational for all $n$. To do this, recall that a polynomial is just a sum of monomials and since the integral of a sum is just the sum of integrals, we can just consider the integral of a monomial over $Q$. Note that in the following calculation $p_{l} \leq q_{l}$ for all $l$ and $\left(q_{1}, \ldots, q_{d}\right)$ and $\left(p_{1}, \ldots, p_{d}\right)$ are diagonal vertices of $Q$.

$$
\begin{aligned}
\int_{Q} a \cdot x_{1}^{k 1} \cdot x_{2}^{k 2} \cdot \ldots \cdot x_{d}^{k d} & =\int_{p_{d}}^{q_{d}} \int_{p_{d-1}}^{q_{d-1}} \ldots \int_{p_{1}}^{q_{1}} a \cdot x_{1}^{k 1} \cdot x_{2}^{k 2} \cdot \ldots \cdot x_{d}^{k d} \\
& =\frac{a\left(q_{1}^{k_{1}+1}-p_{1}^{k_{1}+1}\right)\left(q_{2}^{k_{2}+1}-p_{2}^{k_{2}+1}\right) \ldots\left(q_{d}^{k_{d}+1}-p_{d}^{k_{d}+1}\right)}{\left(k_{1}+1\right)\left(k_{2}+1\right) \ldots\left(k_{d}+1\right)}
\end{aligned}
$$

This is a rational number because by the definition of $f_{n}, a$ must be rational and $q_{1}, p_{1}, \ldots, q_{d}, p_{d}$ are also rational because they are the coordinates of our rational cube $Q$. Thus, we see that $\int_{Q} f_{n}$ is rational for all $n$. Also, since $\left(f_{n}\right)$ is a recursive sequence, $\left(\int_{Q} f_{n}\right)$ is also a recursive sequence. We say this because $\int_{Q} f_{n}$ can be re-written as the integral over $Q$ of the elements of the sequence $\left(f_{n}\right)$. Now that we have our recursive 
sequence of rational numbers, we just need to show that $\left(\int_{Q} f_{n}\right)$ converges to $\int_{Q} f$ at a nice rate.

$$
\begin{aligned}
\left|\int_{Q} f_{n}-\int_{Q} f\right| & \leq \int_{Q}\left|f_{n}-f\right| \\
& \leq \int_{[0,1]^{d}}\left|f_{n}-f\right| \\
& \leq \frac{1}{2^{n}}
\end{aligned}
$$

This proves that $\int_{Q} f$ exists and is computable.

We now define $\Sigma_{1}^{0}$ sets.

Definition 2.4 A set $S \subseteq \mathbb{N}^{k} \times[0,1]^{d}$ is called $\Sigma_{1}^{0}$ if there exists a recursive predicate $R \subseteq \mathbb{N}^{k+1} \times(\mathbb{Q} \cap[0,1])^{2 d}$ such that

$$
\begin{aligned}
S= & \left\{\left\langle m_{1}, \ldots, m_{k}, x_{1}, \ldots, x_{d}\right\rangle:(\exists j \in \mathbb{N})\left(\exists a_{1}, b_{1}, \ldots, a_{d}, b_{d} \in \mathbb{Q}\right)\right. \\
& \left.\left(R\left(j, m_{1}, \ldots, m_{k}, a_{1}, b_{1}, \ldots, a_{d}, b_{d}\right) \wedge a_{1}<x_{1}<b_{1} \wedge \ldots \wedge a_{d}<x_{d}<b_{d}\right)\right\} .
\end{aligned}
$$

Definition 2.5 A sequence of sets $\left(U_{n}\right) \subseteq[0,1]^{d}$ is called uniformly $\Sigma_{1}^{0}$ if the predicate $S \subseteq \mathbb{N} \times[0,1]^{d}$ where

$$
S(n, x) \equiv x \in U_{n}
$$

is $\Sigma_{1}^{0}$

The next proposition provides a useful property of $\Sigma_{1}^{0}$ sets.

Proposition 2.6 The class of $\Sigma_{1}^{0}$ sets is closed under the existential number quantifier.

The next definition was first given by Martin-Löf [3].

Definition 2.7 A point $x \in \mathbb{R}^{d}$ is Martin-Löfrandom if $x$ does not lie in the intersection of any uniformly $\Sigma_{1}^{0}$ sequence $\left(V_{k}\right)$ such that $\mu\left(V_{k}\right) \leq \frac{1}{2^{k}}$ for each $k$.

Another characterization of random points in $\mathbb{R}^{d}$ is given by Solovay's Lemma, as proven by Simpson [5]. The proof given by Simpson [5] is given for sets in the Cantor space, but the proof applies here as well. 
Lemma 2.8 (Solovay's Lemma) Suppose that $\left(V_{k}\right)$ is a sequence of uniformly $\Sigma_{1}^{0}$ sets in $[0,1]^{d}$ such that

$$
\sum_{k=1}^{\infty} \mu\left(V_{k}\right)<\infty
$$

Then for any random $x \in[0,1]^{d}, x$ lies in only finitely many $V_{k}$.

Before we begin the proof of our modified Lebesgue Differentiation Theorem, we will need a few concepts and results to help set up the proof of the theorem.

\section{A canonical representative of $f$}

Lemma 3.1 (Chebyshev Inequality) Given an $L_{1}$-computable function $f$, and $\varepsilon>0$, let

$$
S(f, \varepsilon)=\{x:|f(x)|>\varepsilon\} .
$$

Then

$$
\mu(S(f, \varepsilon)) \leq \frac{\|f\|_{1}}{\varepsilon}
$$

Proof Consider $\|f\|_{1}$.

$$
\left|f \|_{1}=\int_{[0,1]^{d}}\right| f\left|\geq \int_{S(f, \varepsilon)}\right| f \mid \geq \int_{S(f, \varepsilon)} \varepsilon \geq \varepsilon \cdot \mu(S(f, \varepsilon))
$$

The result follows.

The next lemma is based on Proposition 4.1 found in a paper by Brown, Guisto and Simpson [1].

Lemma 3.2 Let $f$ be $L_{1}$-computable. Then, there exists a uniformly $\Sigma_{1}^{0}$ sequence of sets $V_{k}, k \in \mathbb{N}$ such that $\mu\left(V_{k}\right) \leq \frac{1}{2^{k-3}}$, and for all $x \notin V_{k}$ and $n \geq k$ we have

$$
\left|f_{i}(x)-f_{2 n}(x)\right| \leq \frac{1}{2^{n}}
$$

for all $i \geq 2 n$. 
Proof Let $f_{n}$ and $D_{n}$ be as in Theorem 2.1. Let $V_{k}=\left\{x \mid(\exists n \geq k)(\exists i \geq 2 n)\left(\mid f_{i}(x)-\right.\right.$ $\left.\left.f_{2 n}(x) \mid>\frac{1}{2^{n}}\right)\right\}$. We want to show that the $V_{k}$ are $\Sigma_{1}^{0}$. Since $f_{n}$ is continuous for all $n$, $x \in V_{k}$ if and only if there exists a ball around $x$ contained in $V_{k}$. For this reason, we can rewrite $V_{k}$ as follows,

$$
\begin{aligned}
x \in V_{k} \equiv & (\exists n \geq k)(\exists i \geq 2 n)(\exists m \in \mathbb{N})\left(\exists a_{1}, b_{1}, \ldots, a_{d}, b_{d} \in \mathbb{Q}\right) \text { such that } \\
& \left(a_{1}<x_{1}<b_{1}, \ldots, a_{d}<x_{d}<b_{d}\right) \wedge\left(\left|f_{i}(a)-f_{2 n}(a)\right|>\frac{1}{2^{n}}+\frac{1}{2^{m}}\right) \wedge \\
& \left(\left(D_{i}+D_{2 n}\right) \cdot|a-b|<\frac{1}{2^{m}}\right)
\end{aligned}
$$

Here $n$ and $i$ are natural numbers and $a=\left\langle a_{1}, \ldots, a_{d}\right\rangle$ and $b=\left\langle b_{1}, \ldots, b_{d}\right\rangle$. Define the predicate $R$ by:

$$
\begin{aligned}
R\left(i, a_{1}, b_{1}, \ldots, a_{d}, b_{d}\right)= & \left(\left|f_{i}(a)-f_{2 n}(a)\right|>\frac{1}{2^{n}}+\frac{1}{2^{m}}\right) \wedge \\
& \left(\left(D_{i}+D_{2 n}\right) \cdot|a-b|<\frac{1}{2^{m}}\right)
\end{aligned}
$$

is a recursive predicate, so by Theorem 2.4 and Theorem 2.5 and Theorem 2.6, we can see that $V_{k}$ is $\Sigma_{1}^{0}$ and the sequence $\left(V_{k}\right)$ is uniformly $\Sigma_{1}^{0}$.

Now, we need to look at the measure of $V_{k}$. Note that

$$
V_{k} \subseteq \bigcup_{n=k}^{\infty} S\left(\sum_{i=2 n}^{\infty}\left|f_{i+1}(x)-f_{i}(x)\right|, \frac{1}{2^{n}}\right)
$$

Using the previous lemma, we can conclude the proof as follows: 


$$
\begin{aligned}
\mu\left(V_{k}\right) & \leq \mu\left(\bigcup_{n=k}^{\infty} S\left(\sum_{i=2 n}^{\infty}\left|f_{i+1}(x)-f_{i}(x)\right|, \frac{1}{2^{n}}\right)\right) \\
& \leq \sum_{n=k}^{\infty} \mu\left(S\left(\sum_{i=2 n}^{\infty}\left|f_{i+1}(x)-f_{i}(x)\right|, \frac{1}{2^{n}}\right)\right) \\
& =\sum_{n=k}^{\infty} 2^{n} \sum_{i=2 n}^{\infty}|| f_{i+1}-f_{i} \|_{1} \\
& \leq \sum_{n=k}^{\infty} 2^{n} \sum_{i=2 n}^{\infty} \frac{1}{2^{i-1}} \\
& \leq \sum_{n=k}^{\infty} \frac{2^{n}}{2^{2 n-2}} \\
& =\sum_{n=k}^{\infty} \frac{1}{2^{n-2}} \\
& =\frac{1}{2^{k-3}}
\end{aligned}
$$

Thus we have our $V_{k}$ and by the definition of $V_{k}$, for all $x$ not in $V_{k}$ and $n \geq k$,

$$
\left|f_{i}(x)-f_{2 n}(x)\right| \leq \frac{1}{2^{n}}
$$

for all $i \geq n$

Lemma 3.3 Let $f$ be $L_{1}$-computable. Then $\lim _{n \rightarrow \infty} f_{n}(x)$ exists for all random $x$.

Proof From the previous lemma, we can see that

$$
\sum_{k=1}^{\infty} \mu\left(V_{k}\right)<\infty .
$$

Since the sum is finite, we can use Solovay's Lemma and say that any random $x$ will only be in finitely many $V_{k}$. So, for some large $k$ and $\forall n \geq k$, we can see that

$$
\left|f_{i}(x)-f_{2 n}(x)\right| \leq \frac{1}{2^{n}}
$$

for all $i \geq 2 n$. From this we can see that $f_{n}$ converges uniformly for $x \notin V_{k}$, and the limit exists. 
Definition 3.4 Let $f \in L_{1}\left([0,1]^{d}\right)$. We define $\widehat{f}(x):[0,1]^{d} \rightarrow \mathbb{R}$ to be

$$
\widehat{f}(x)=\left\{\begin{array}{l}
\lim _{n \rightarrow \infty} f_{n}(x) \text { if } x \text { is random } \\
0 \text { otherwise }
\end{array}\right.
$$

By Theorem $3.3 \lim _{n \rightarrow \infty} f_{n}(x)$ exists for all random $x$ so we know our new function is well-defined. We want to claim that $\widehat{f}$ is a canonical representation of the equivalence class of $f\left(f \sim g \Leftrightarrow \mid f f-g \|_{1}=0\right)$. The next two lemmas will prove that this is indeed the case.

\section{Lemma $3.5 \int_{[0,1]^{d}}|f-\widehat{f}| d x=0$}

Proof Let $E_{\varepsilon}=\{x:|f(x)-\widehat{f}(x)|>\varepsilon\}$ and consider the set $E_{0}$. If this is a set of measure zero, then the result follows. Suppose, however, that this is not the case. Then, there exists some small $\varepsilon>0$ such that $\mu\left\{E_{\varepsilon}\right\}>\varepsilon$. Now, by Theorem 3.2, for all random $x$, there exists $k$ large such that $x \notin V_{k}$ and for all $n \geq k,\left|f_{i}(x)-f_{2 n}(x)\right| \geq \frac{1}{2^{n}}$ for all $i \geq k$. By the definition of $\widehat{f}$ we can also say that $\left|\widehat{f}(x)-f_{2 n}(x)\right| \geq \frac{1}{2^{n}}$. So for $n$ such as the one above and $x \notin V_{k}$,

$$
\begin{aligned}
\mu\left\{x:\left|f(x)-f_{2 n}(x)\right|>\varepsilon-\frac{1}{2^{n}}\right\} & >\mu\left\{x:|\widehat{f}(x)-f(x)|-\left|\widehat{f}(x)-f_{2 n}(x)\right|>\varepsilon-\frac{1}{2^{n}}\right\} \\
& >\mu\left\{x:|\widehat{f}(x)-f(x)|-\frac{1}{2^{n}}>\varepsilon-\frac{1}{2^{n}}\right\} \\
& =\mu\{x:|\widehat{f}(x)-f(x)|>\varepsilon\} \\
& >\varepsilon-\frac{1}{2^{n-3}}
\end{aligned}
$$

By Theorem 3.1,

$$
\left\|f-f_{2 n}\right\|_{1} \geq\left(\varepsilon-\frac{1}{2^{n}}\right) \cdot \mu\left\{x:\left|f(x)-f_{2 n}(x)\right|>\varepsilon-\frac{1}{2^{n}}\right\}>\left(\varepsilon-\frac{1}{2^{n}}\right)\left(\varepsilon-\frac{1}{2^{n-3}}\right) .
$$

This is a contradiction because $\left\|f-f_{2 n}\right\|_{1} \rightarrow 0$ at $n \rightarrow \infty$. It follows that our assumption is incorrect and therefore, $E_{0}$ has measure zero.

Lemma 3.6 Given two $L_{1}$-computable functions, $f, g$

$$
\int_{[0,1]^{d}}|f-g|=0 \text { iff } \widehat{f}=\widehat{g} .
$$


Proof $(\Leftarrow)$ Suppose $\widehat{f}=\widehat{g}$. Then, $f(x)=g(x)$ for all $x$ except for a set of measure zero. The result follows.

$(\Rightarrow)$ Suppose $\int_{[0,1]^{d}}|f-g|=0$. Then,

$$
\begin{aligned}
\left\|f_{n}-g_{n}\right\|_{1} & =\left\|f_{n}-f+f-g+g-g_{n}\right\|_{1} \\
& \leq\left\|f_{n}-f\right\|_{1}+\|f-g\|_{1}+\left\|g-g_{n}\right\|_{1} \\
& \leq \frac{2}{2^{n}}
\end{aligned}
$$

This is a useful fact that will be used a little later. First we look at another consequence of our given assumption.

$$
\begin{aligned}
\int_{[0,1]^{d}}|f-g| & =\int_{[0,1]_{\text {random }}^{d}}|f-g| \\
& =\int_{[0,1]^{d}}|\widehat{f}-\widehat{g}|
\end{aligned}
$$

This means that $\widehat{f}=\widehat{g}$ except on a set of measure $0, E$.

$$
E=\{x \mid \widehat{f} \neq \widehat{g}\}=\left\{x \mid \lim _{n \rightarrow \infty}\left(f_{n}(x)-g_{n}(x)\right) \neq 0\right\} .
$$

We would like to show that there cannot be any random $x \in E$. Let

$$
V_{n}^{k}=\left\{x:\left|f_{n}(x)-g_{n}(x)\right|>\frac{1}{2^{k}}\right\}
$$

The sets $V_{n}^{k}$ will be our test for randomness. Using sets similar to the ones used in the proof of Theorem 3.2, we can show that for a fixed $k$ the sequence $\left(V_{n}^{k}\right)$ is uniformly $\Sigma_{1}^{0}$.

We would now like to use Solovay's Lemma. To do that, we need to show that the sum of the measures of $V_{n}^{k}$ over all $n$ is finite. To do this, we will use Theorem 3.1 again.

$$
\begin{aligned}
\mu\left(V_{n}^{k}\right) & =\mu\left(x:\left|f_{n}(x)-g_{n}(x)\right|>\frac{1}{2^{k}}\right) \\
& \leq 2^{k} \cdot\left\|f_{n}-g_{n}\right\|_{1} \\
& \leq \frac{2^{k}}{2^{n-1}}
\end{aligned}
$$

For a fixed $k$ this is a geometric series, so $\sum_{n=1}^{\infty}\left|V_{n}^{k}\right|<\infty$. By Solovay's Lemma, for any fixed $k, x$ can only be in finitely many $V_{n}^{k}$. Therefore for a random $x$, $\lim _{n \rightarrow \infty}\left|f_{n}(x)-g_{n}(x)\right| \leq \frac{1}{2^{k}}$ for all $\mathrm{k}$ meaning that $\lim _{n \rightarrow \infty}\left|f_{n}(x)-g_{n}(x)\right|=0$. This shows that there cannot be a random $x$ in E. By the definition of $\widehat{f}$ and $\widehat{g}$, there cannot be a non-random $x$ in $E$ either. This means that $E$ is empty, and $\widehat{f}=\widehat{g}$ 
From the last two lemmas, we can see that $\widehat{f}$ is a canonical representative of the equivalence class of $f$. The next section provides some results necessary for the proof of the main theorem.

\section{Some Important Lemmas}

First, will will prove the Lebesgue Differentiation theorem for continuous functions.

Proposition 4.1 Let $f \in L_{1}\left(\mathbb{R}^{d}\right)$ be a continuous function. Then, the indefinite integral of $f$ is differentiable and its derivative is equal to $f(x)$ for all $x \in \mathbb{R}^{d}$.

Proof The proof is clear from the following calculations. If $f$ is continuous at $x$ and $Q$ is a rational cube containing $x$, then

$$
\begin{aligned}
\left|\frac{1}{\mu(Q)} \int_{Q} f(y) d y-f(x)\right| & =\left|\frac{1}{\mu(Q)} \int_{Q}[f(y)-f(x)] d y\right| \\
& \leq \frac{1}{\mu(Q)} \int_{Q}|f(y)-f(x)| d y \\
& \leq \sup _{y \in Q}|f(y)-f(x)|,
\end{aligned}
$$

which tends to zero as $Q$ shrinks to $x$.

Using this fact we develop the idea for the proof. By Theorem 2.1, we can approximate our function $f$ using continuous polynomials. Using this, we can approximate the indefinite integral of $f$ and create a test for randomness. To do all this, we will need a few lemmas.

Lemma 4.2 (Simple Vitali Lemma) Let $E$ be a subset of $[0,1]^{d}$, and let $K$ be a collection of cubes $Q$ in $[0,1]^{d}$ covering $E$. Then there exists a positive constant $\beta$, depending only on $d$, and a finite number of disjoint cubes $Q_{1}, \ldots, Q_{N}$ in $K$ such that

$$
\sum_{j=1}^{N} \mu\left(Q_{j}\right) \geq \beta \cdot \mu(E)
$$

A proof of the Simple Vitali Lemma is given by Wheedon and Zygmund [6] on page 102. 
Definition 4.3 Consider a function $g:[0,1]^{d} \rightarrow \mathbb{R}$ that is integrable over every cube $Q \subseteq[0,1]^{d}$. Let

$$
g^{*}(x)=\sup \left\{\frac{1}{\mu(Q)} \int_{Q}|g(y)| d y\right\}
$$

where the supremum is taken over every cube $Q$ with center $x$ and edges parallel to the coordinate axes. This function, $g^{*}$ is called the Hardy-Littlewood maximal function of $g$.

This is a slightly modified version of the definition of the Hardy-Littlewood maximal function.

Definition 4.4 Consider an $L_{1}$ function $g:[0,1]^{d} \rightarrow \mathbb{R}$. We say $g$ belongs to weak $L\left([0,1]^{d}\right)$ if there exists $c$ independent of $\alpha$ such that

$$
\mu\left\{x \in[0,1]^{d}:|g(x)|>\alpha\right\} \leq \frac{c}{\alpha} \quad(\alpha>0) .
$$

Lemma 4.5 (Hardy-Littlewood) Given an $L_{1}$ function $f:[0,1]^{d} \rightarrow \mathbb{R}, f^{*}$ belongs to weak $L\left([0,1]^{d}\right)$. Moreover, there is a constant $c$ independent of $f$ and $\alpha$ such that

$$
\mu\left\{x \in[0,1]^{d}: f^{*}(x)>\alpha\right\} \leq \frac{c}{\alpha} \int_{[0,1]^{d}}|f|, \alpha>0
$$

Proof Since the domain of $f$ and $f^{*}$ is $[0,1]^{d}$ if we fix $\alpha>0$ we can say that the measure of the set

$$
E=\left\{x: f^{*}(x)>\alpha\right\}
$$

is finite. If $x \in E$, there is a rational cube $Q_{x}$ containing $x$ such that $\frac{1}{\mu\left(Q_{x}\right)} \int_{Q_{x}}|f|>\alpha$, or

$$
\mu\left(Q_{x}\right)<\frac{1}{\alpha} \int_{Q_{x}}|f|
$$

The collection of such $Q_{x}$ covers $E$, so by Theorem 4.2, there exist $\beta>0$ and $x_{1}, \ldots x_{N} \in E$ such that $Q_{x_{1}}, \ldots, Q_{x_{N}}$ are disjoint and $\mu(E)<\frac{1}{\beta} \sum_{j=1}^{N} \mu\left(Q_{x_{j}}\right)$. Putting everything together, we get

$$
\mu(E)<\frac{1}{\beta} \sum_{j=1}^{N} \frac{1}{\alpha} \int_{Q_{x_{j}}}|f|=\frac{1}{\beta \alpha} \int_{\bigcup_{j=1}^{N} Q_{x_{j}}}|f| \leq \frac{1}{\beta \alpha} \int_{[0,1]^{d}}|f| .
$$

This proves the Hardy-Littlewood Lemma. 
Definition 4.6 Given $f \in L_{1}$ and $\varepsilon>0$, let $S^{*}(f, \varepsilon)$ be the union of all $Q$ such that

$$
\frac{\int_{Q}|f|}{\mu(Q)}>\varepsilon
$$

Note that according to this definition, and the Hardy-Littlewood Lemma,

$$
\mu\left(S^{*}(f, \varepsilon)\right) \leq \frac{c|| f \|_{1}}{\varepsilon} .
$$

Lemma 4.7 Let $c$ be the constant from the Hardy-Littlewood Lemma and let $f$ be $L_{1}$-computable. Then, we can find sets $V_{k}^{*}$ which are uniformly $\Sigma_{1}^{0}$ such that $\mu\left(V_{k}^{*}\right) \leq \frac{c}{2^{k-1}}$ and for all $x \notin V_{k}^{*}$ and $n \geq k$ we have that

$$
\frac{\int_{Q}\left|f-f_{2 n}\right|}{\mu(Q)} \leq \frac{1}{2^{n}}
$$

for all $Q$ containing $x$.

Proof Let

$$
V_{k}^{*}=\bigcup_{n=k}^{\infty} S^{*}\left(f-f_{2 n}, \frac{1}{2^{n}}\right)
$$

Since the union of countable many uniformly $\Sigma_{1}^{0}$ sets is $\Sigma_{1}^{0}$, we need to show that the sequence $\left(S^{*}\left(f-f_{2 n}, \frac{1}{2^{n}}\right)\right)$ is uniformly $\Sigma_{1}^{0}$. Let

$$
R_{n}\left(\varepsilon, a_{1}, b_{1}, \ldots, a_{d}, b_{d}\right)=\frac{\int_{a_{d}}^{b_{d}} \ldots \int_{a_{1}}^{b_{1}} f(x)}{\left(a_{1}-b_{1}\right) \cdot \ldots \cdot\left(a_{d}-b_{d}\right)}>\varepsilon .
$$

Then, we can write $S^{*}\left(f-f_{2 n}, \frac{1}{2^{n}}\right)$ in the following way:

$$
\begin{aligned}
S^{*}\left(f-f_{2 n}, \frac{1}{2^{n}}\right)= & \left\{x: \exists a_{1}, b_{1}, \ldots, a_{d}, b_{d} \in \mathbb{Q}_{[0,1]} \mid\left(R\left(\frac{1}{2^{n}}, a_{1}, b_{1}, \ldots, a_{d}, b_{d}\right)\right)\right. \\
& \text { and } \left.a_{1}<x_{1}<b_{1}, \ldots, a_{d}<x_{d}<b_{d}\right\}
\end{aligned}
$$

This is a sequence of uniformly $\Sigma_{1}^{0}$ sets by Theorem 2.5 which means that each $V_{k}^{*}$ is $\Sigma_{1}^{0}$ and the sequence $\left(V_{k}^{*}\right)$ is uniformly $\Sigma_{1}^{0}$. Now we need to look at the measure of 
$V_{k}^{*}$. We will use the Hardy-Littlewood Lemma.

$$
\begin{aligned}
\mu\left(V_{k}^{*}\right) & \leq \sum_{n=k}^{\infty} \mu\left(S^{*}\left(f-f_{2 n}, \frac{1}{2^{n}}\right)\right) \\
& \leq \sum_{n=k}^{\infty} c \cdot 2^{n} \cdot\left\|f-f_{2 n}\right\|_{1} \\
& \leq \sum_{n=k}^{\infty} \frac{c 2^{n}}{2^{2 n}} \\
& \leq \sum_{n=k}^{\infty} \frac{c}{2^{n}} \\
& =\frac{c}{2^{k-1}}
\end{aligned}
$$

The result follows.

There is one last lemma we need before we can prove the main result.

Lemma 4.8 Let $f$ be $L_{1}$-computable and let $D_{n}$ and $f_{n}$ be as in Theorem 2.1. Then for all $k, n \geq k$ and all $x \notin V_{k} \cup V_{k}^{*}$,

$$
\left|\widehat{f}(x)-\frac{\int_{Q} f}{\mu(Q)}\right| \leq \frac{1}{2^{n-1}}+D_{2 n} \cdot(\text { diameter of } Q)
$$

for all rational cubes $Q$ containing $x$. Here the sequence of $V_{k}$ is from Theorem 3.2 and the sequence of $V_{k}^{*}$ is from Theorem 4.7.

Proof Since $D_{n}$ is an upper bound of the maximum gradient of each $f_{n}, \max \left\{\left|\nabla f_{n}\right|\right.$ : $\left.x \in[0,1]^{d}\right\}$, we can use the Mean Value Theorem to say,

$$
\left|f_{2 n}(x)-\frac{\int_{Q} f_{2 n}}{\mu(Q)}\right| \leq D_{2 n} \cdot(\text { diameter of } \mathrm{Q})
$$

for all rational $Q$ containing $x$. By Theorem 3.2 we have that

$$
\left|\widehat{f}(x)-f_{2 n}(x)\right| \leq \frac{1}{2^{n}}
$$

and by Theorem 4.7,

$$
\frac{\int_{Q}\left|f-f_{2 n}\right|}{\mu(Q)} \leq \frac{1}{2^{n}}
$$

Journal of Logic \& Analysis 1:9 (2009) 
for all $n \geq k$ and $x \notin V_{k} \cup V_{k}^{*}$. Combining these two, we get that

$$
\begin{aligned}
\left|\widehat{f}(x)-\frac{\int_{Q} f}{\mu(Q)}\right| & =\left|\widehat{f}(x)-f_{2 n}(x)+f_{2 n}(x)-\frac{\int_{Q} f_{2 n}}{\mu(Q)}+\frac{\int_{Q} f_{2 n}}{\mu(Q)}-\frac{\int_{Q} f}{\mu(Q)}\right| \\
& \leq\left|\widehat{f}(x)-f_{2 n}(x)\right|+\left|\frac{\int_{Q} f_{2 n}}{\mu(Q)}-\frac{\int_{Q} f}{\mu(Q)}\right|+\left|f_{2 n}(x)-\frac{\int_{Q} f_{2 n}}{\mu(Q)}\right| \\
& \leq \frac{1}{2^{n}}+\frac{1}{2^{n}}+D_{2 n} \cdot(\text { diameter of Q) } \\
& \leq \frac{1}{2^{n-1}}+D_{2 n} \cdot(\text { diameter of Q) }
\end{aligned}
$$

\section{Main Result}

Theorem 5.1 Let $f$ be an $L_{1}$-computable function. Let $\widehat{f}$ be the canonical representation of $f$ as defined in Theorem 3.4. Then for all random $x$,

$$
\widehat{f}(x)=\lim _{Q \searrow x} \frac{\int_{Q} f}{\mu(Q)}
$$

for $Q$ containing $x$.

Proof Let $f_{n}$ and $D_{n}$ be as in Theorem 2.1. $V_{k}$ from Theorem 3.2 and $V_{k}^{*}$ from Theorem 4.7 form Martin-Löf tests. So, for a random $x$, there exists a large $k$ such that $x \notin V_{k} \cup V_{k}^{*}$. We want to show that for all $\varepsilon>0 \exists \delta>0$ such that

$$
\left|\widehat{f}(x)-\frac{\int_{Q}|f|}{\mu(Q)}\right|<\varepsilon
$$

whenever the diameter of $Q$ is less than $\delta$. Choose $n$ large so that $\frac{1}{2^{n-1}}<\frac{\varepsilon}{2}$ and let $\delta=\frac{\varepsilon}{2 D_{2 n}}$. Then, when the diameter of $Q$ is less than $\delta$,

$$
\left|\widehat{f}(x)-\frac{\int_{Q}|f|}{\mu(Q)}\right|<\frac{1}{2^{n-1}}+\frac{\varepsilon}{2}<\varepsilon .
$$




\section{Closing Remarks}

By only dealing with $L_{1}$-computable functions, our theorem seems at first to be less general than the original Lebesgue Differentiation Theorem. However, if we consider relativization, it can be seen that the statement proved in this paper is stronger. Any $L_{1}$ function is computable relative to some oracle and using this we can prove a relativized version of Theorem 5.1 pertaining to any function and provide a very specific set of measure zero, outside of which the Lebesgue Differentiation Theorem always holds.

In Theorem 5.1 we have proved that the Lebesgue Differentiation Theorem holds at $x$ provided that $x$ is a Martin-Löf random point in a Euclidean space. The natural question arises, is the converse true? That is, if we have that the Lebesgue Differentiation Theorem holds at $x$ for all $L_{1}$-computable functions, is $x$ necessarily random? This is an important question as the converse holding would give an alternative characterization of random points in Euclidean space.

\section{References}

[1] D K Brown, M Giusto, S Simpson, Vitali's Theorem and WWKL, Archive for Mathematical Logic 41 (2002), 191-206; doi: 10.1007/s001530100100.

[2] O Demuth, The differentiability of constructive functions of weakly bounded variation on pseudo numbers., Comment. Math. Univ. Carolinae 16 (1975), 583-599.

[3] P Martin-Löf, The definition of random sequences,Information and Control 9 (1966) 602-619, doi: 10.1016/S0019-9958(66)80018-9.

[4] M B Pour-EI, J I Richards, Computability in Analysis and Physics, Springer-Verlag, 1989.

[5] S Simpson, Almost everywhere domination and superhighness, Mathematical Logic Quaterly 53 (2007) 462-482; doi: 10.1002/malq.200710012.

[6] R Wheeden, A Zygmund, Measure and Integral, Marcel Dekker, Inc, 1977.

The Pennsylvania State University, Department of Mathematics, McAllister Building, University Park, PA 16802, USA

pathak@math.psu.edu

Received: 23 September $2008 \quad$ Revised: 13 July 2009 